\title{
Structures and intermittency in a passive scalar model
}

\author{
M. Vergassola ${ }^{1}$ and A. Mazzino ${ }^{2}$ \\ ${ }^{1}$ CNRS, Observatoire de Nice, B.P. 4229, 06304 Nice Cedex 4, France. \\ ${ }^{2}$ INFM - Dipartimento di Fisica, Università di Genova, I-16146 Genova, Italy.
}

October 31, 2018

\begin{abstract}
A one-dimensional white-in-time passive scalar model is introduced. Strong and persistent structures are shown to be present. A perturbative expansion for the scaling exponents is performed around a Gaussian limit of the model. The resulting predictions are compared with numerical simulations.
\end{abstract}

PACS number(s) : 47.10.+g, 47.27.-i, 05.40.+j

It is commonly observed in turbulent flows that coherent and well localized structures emerge in the sea of disorganized, random regions. Well-known examples are provided by 2D and 3D Navier-Stokes turbulence: numerical simulations show that structures appear respectively in the form of quasi-circular vortices and thin, elongated vortex tubes. No ab initio theory for the statistical properties of the flows (and a fortiori of their dependence on the structures) is however available. A systematic statistical theory has recently been developed for white-in-time passive field models. Their crucial property is that simultaneous correlation functions obey closed equations of motion [1]. Anomalous scaling and intermittency have in particular been considered. The general mechanism for the appearance of anomalous scaling is associated with zero modes of the equations for the correlation functions [2, 3, 4]. For Kraichnan passive scalar model, anomalies first appear at the level of fourth-order correlations $[1-3,5]$. When a large-scale gradient is imposed, also the third-order moment is anomalous [6]. Scaling exponents have been calculated by using perturbative expansions around Gaussian limits [2, 3]. For magnetic fields a nonperturbative anomalous solution for second-order correlations has been found [7]. The zero mode responsible for anomalous scaling comes from the balance in the inertial range between field lines stretching and eddy-damping. This suggests that also for white-in-time models, structures could be present and play an important role. A crucial 
point is whether the perturbative expansions around Gaussian limits are capable, as those in critical phenomena, to correctly capture the global statistical effects of structures. Comparing the perturbative predictions with the results of numerical simulations would clearly help clarifying this issue. The $\delta$-correlation in time of the advecting velocity makes however simulations quite intricate [8]. We have therefore concentrated our attention on the 1D white-in-time passive scalar model presented here. As in Kraichnan model, the velocity is Gaussian and $\delta$-correlated in time. Its structure function scales with a positive exponent $\xi$ and the dynamics has a Gaussian limit for $\xi \rightarrow 0$.

The simulations show that the activity of the scalar is indeed concentrated in strongly localized peaks. Despite of the $\delta$-correlation in time of the advecting velocity, their lifetime can be significant. The mechanism of formation of the peaks is associated with the stretching due to velocity gradients. The peaks are responsible for the observed very high tails of the probability distribution functions (p.d.f.).

The $\delta$-correlation in time ensures that correlation functions satisfy closed equations of motion. The behaviour of second-order correlations is obtained analytically and their scaling is normal. Predictions for the scaling exponents of higher-order correlations are derived using the perturbation scheme in $\xi$ proposed in Ref. [3]. Analytic predictions (in the form of Padé approximants) for the fourth and sixth-order structure functions are compared for $\xi=0.5$ with numerical results.

The equation of the model is

$$
\partial_{t} \phi+\nabla(v \phi)=\kappa \Delta \phi+f .
$$

Here, both the velocity $v$ and the injection $f$ are Gaussian and $\delta$-correlated in time. The velocity correlation function is

$$
\left\langle v(x, t) v\left(x^{\prime}, t^{\prime}\right)\right\rangle=\delta\left(t-t^{\prime}\right)\left[D_{0}-S\left(\left|x-x^{\prime}\right|\right)\right] .
$$

The structure function $S$ scales as $S\left(x-x^{\prime}\right)=D\left|x-x^{\prime}\right|^{\xi}$ (and $0<\xi<2$ ) in the range of scales between the ultraviolet and the infrared cutoffs $\Lambda_{U V}$ and $\Lambda_{I R}$ (the smallest and the largest scales in our problem). The large-scale injection satisfies $\left\langle f(x, t) f\left(x^{\prime}, t^{\prime}\right)\right\rangle=$ $\delta\left(t-t^{\prime}\right) F_{L}\left(x-x^{\prime}\right)$ with its Fourier transform $\hat{F}_{L}$ concentrated around wave numbers $O(1 / L)$. It is assumed that $\hat{F}_{L}$ vanishes at $k=0$, i.e. $\int_{-\infty}^{+\infty} F_{L}(x) d x=0$. This condition is associated with the fact that $\phi$ is a "gradient field". The equation for the gradients in Kraichnan model indeed coincides with (11) when vector components are omitted. The gradient nature of $\phi$ appears even more clearly from the solution for the second-order correlations presented later. The homogeneous part of the equation for the integrated field $\theta(x, t)=\int^{x} \phi(y, t) d y$ (scaling with positive exponents) is

$$
\partial_{t} \theta+v \nabla \theta=\kappa \Delta \theta
$$




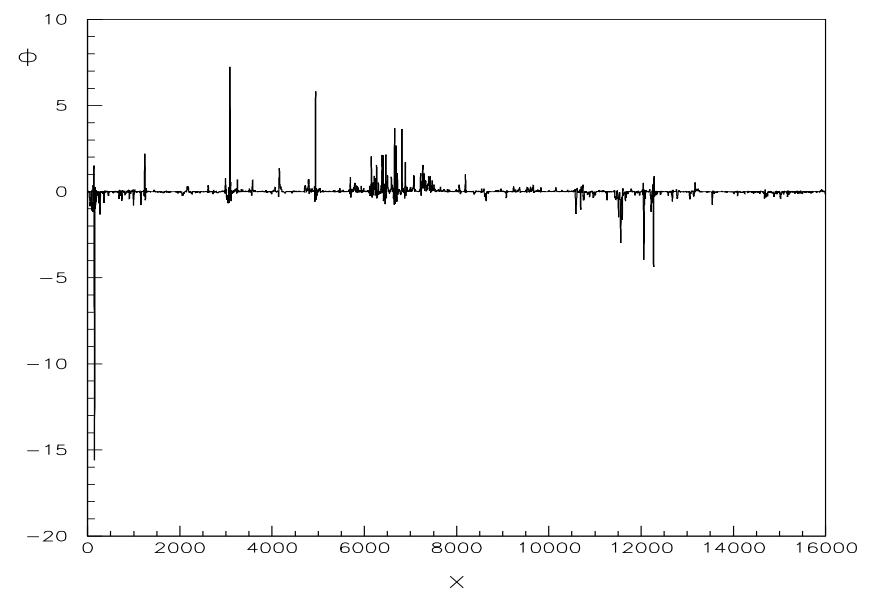

Figure 1: The gradient field $\phi$ vs the spatial coordinate $x$ for $\xi=0.5$.

Note that for (3) the maximum principle holds. The stretching process due to the gradients of $v$ operates on the gradient field $\phi$. To fully resolve the structures we have then preferred to deal directly with (11). This equation has been integrated by using a pseudo-spectral code with periodic boundary conditions. At each time step a new realization of both $v$ and $f$ is generated. The multiplicative term in (11) is numerically treated explicitly (à la Ito). The term differing in the formulations Ito and Stratonovich (the latter being relevant for (11)) is taken into account [9]. The anomalous exponents are not expected to depend on the type of dissipation. For the measurement of the scaling exponents we have then used a $k^{8}$ hyperdissipation, but we have also checked that structures are present for normal dissipation. The resolution is $N=2^{14}$. The injection is concentrated on the first mode. The velocity $v$ is simply generated in Fourier space.

A typical plot of the gradient field $\phi$ is shown in Fig. 1. It is evident the presence of huge peaks of activity. Their mechanism of formation (and persistence) can be immediately grasped from the original equation (11). Neglecting diffusion and forcing, the extrema $\bar{\phi}$ of the field $\phi$ obey $\partial_{t} \bar{\phi}=-(\nabla v) \bar{\phi}$. Since $v$ is $\delta$-correlated in time, the logarithm of $\bar{\phi}$ evolves then as a Brownian motion. On the other hand, it is known that random walkers have a tendency not to change sign. Specifically, let us start a walk from the origin. The cumulative distribution for the fraction of the walk spent on the negative side obeys the arc sine law [10]: keeping the same sign for the whole walk and equipartition of the time between positive and negative values are the most and the least probable events, respectively. In our case, this implies that there will be very long periods of time when the stretching mechanism operates. Peaks such as those in Fig. 1 can then be maintained 


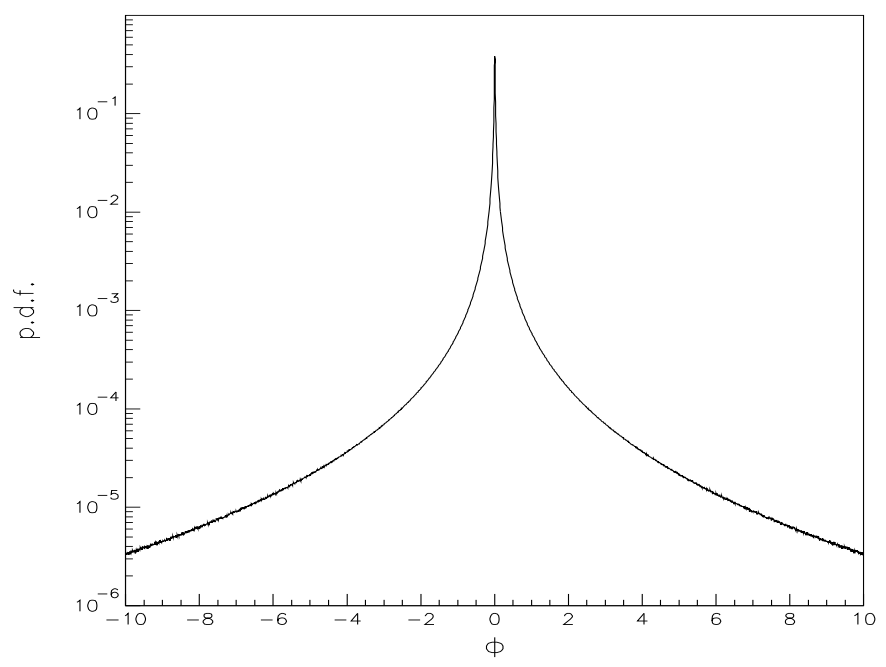

Figure 2: The probability distribution function (p.d.f.) of the gradient field $\phi$, normalized to its r.m.s. value $\sqrt{\left\langle\phi^{2}\right\rangle}$.

for significant times, despite the $\delta$-correlation of the velocity. This is indeed observed in our numerical simulations both for normal and hyper-dissipation. Strong peaks persist for long enough to make them clearly identifiable and meaningful the use of the word "structures" to denote them. The structures are evidently the cause of the high tails of the p.d.f. in Fig. 2.

Let us now derive the predictions for the scaling exponents. The $\delta$-correlation in time ensures that correlation functions obey closed equation of motion. By using Gaussian integration by parts, it is for example easy to derive the equation for the second-order correlation $C_{2}(x, t)=\langle\phi(x, t) \phi(0, t)\rangle$ :

$$
\partial_{t} C_{2}=\frac{d^{2}}{d x^{2}}\left[(2 \kappa+S) C_{2}\right]+F_{L} .
$$

The solution is even and satisfies $\int C_{2}=0$. In the inertial range of scales it decays with the negative exponent $-\xi$. The scaling of the second-order structure function of $\theta$ is obtained by simple integration. For $\xi<1$, its inertial scaling is $2-\xi$, as in Kraichnan model.

Let us now consider the scaling of higher-order correlation functions. Closed equations of motion can be written both for the correlations $C_{2 n}=\left\langle\phi\left(x_{1}\right) \ldots \phi\left(x_{2 n}\right)\right\rangle$ and those of the integrated field $\theta$. Anomalous scaling is associated with the zero modes of the 


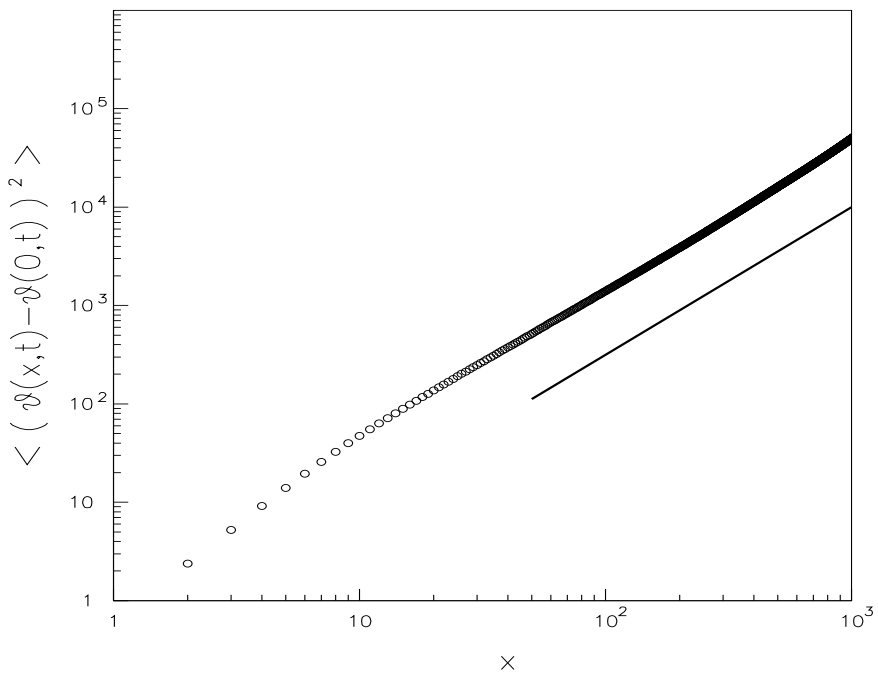

Figure 3: The measured second-order structure function for $\xi=0.5$. The solid line has the slope $2-\xi=1.5$.

homogeneous equations. The relevant equations for the zero mode $Z_{2 n}$ of the $2 n$-th order $\phi$ correlator are

$$
\mathcal{H} Z_{2 n}=\sum_{k \neq j, 1}^{2 n} \nabla_{j} \nabla_{k}\left[S\left(x_{j k}\right) Z_{2 n}\right]=0 .
$$

where $x_{j k}=x_{j}-x_{k}$ and $\nabla_{j}$ stands for the spatial derivative with respect to $x_{j}$. Following Refs. [3] and [11], we look for a scale-invariant solution of this equation as an expansion in powers of $\xi$. Specifically, the structure functions are expanded as $S\left(x_{j k}\right)=$ $D\left(1+\xi \log \left|x_{j k}\right|\right)+O\left(\xi^{2}\right)$ and $Z_{2 n}=Z_{2 n}^{(0)}+\xi Z_{2 n}^{(1)}+O\left(\xi^{2}\right)$. The equations (for any $n$ ) at the lowest order in $\xi$ are trivially satisfied by taking $Z_{2 n}^{(0)}=$ const. $=\bar{Z}_{2 n}$. At the first order in $\xi$, the solution is

$$
Z_{2 n}\left(x_{1}, \ldots, x_{2 n}\right)=\bar{Z}_{2 n}\left[1-\frac{\xi}{2} \sum_{k \neq j, 1}^{2 n} \log \left|x_{j k}\right|\right] .
$$

The scaling exponent (at order $\xi$ ) of the zero mode can now be obtained operating on $Z_{2 n}$ with the Euler operator $\mathcal{E}=\sum_{j=1}^{2 n}\left(x_{j} \nabla_{j}\right)$. It is easily checked that the equality $\mathcal{E} Z_{2 n}=-\xi n(2 n-1) Z_{2 n}$ holds at the first order in $\xi$. The amplitude of the correction to normal scaling $-n \xi$ is thus $2 n(n-1) \xi$. This same value is obtained considering the equation for the correlations of the integrated field $\theta$. Note that the anomalous factor 

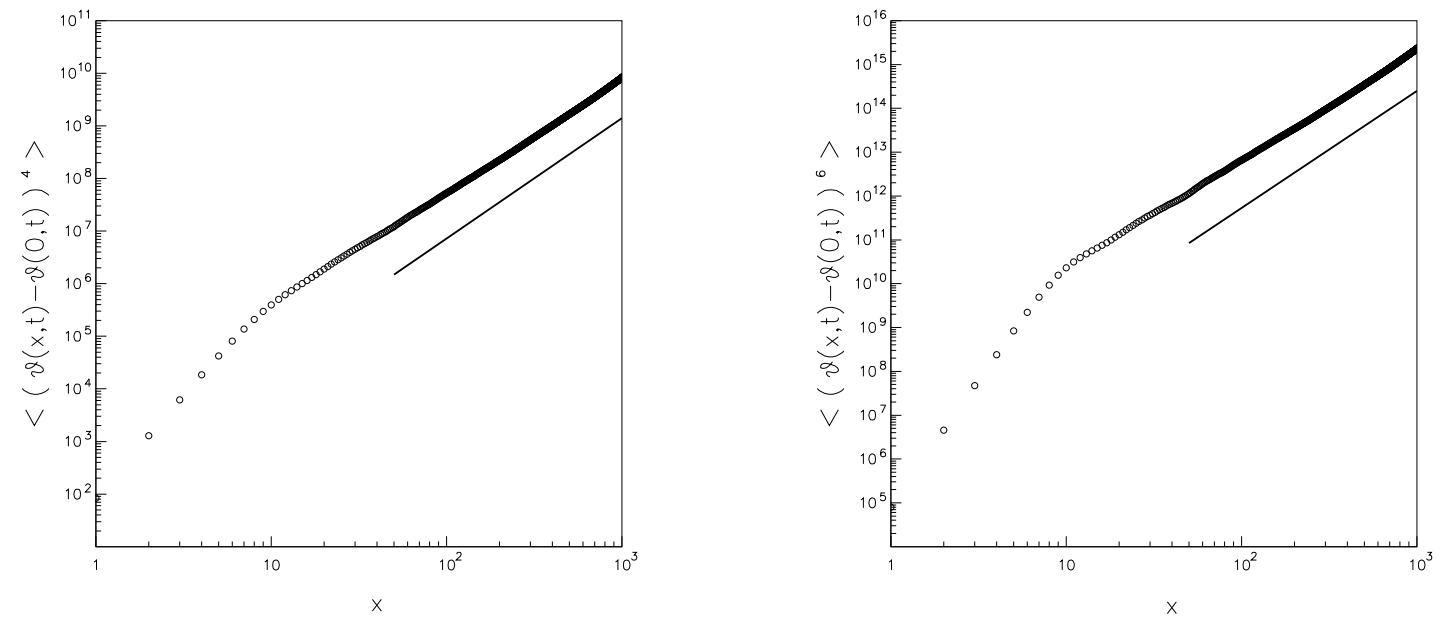

Figure 4: The measured fourth (left) and sixth (right) order structure functions for $\xi=$ 0.5. Solid lines have the slopes predicted by (7), i.e. $\zeta_{4} \simeq 2.29$ and $\zeta_{6} \simeq 2.67$.

$2 n(2 n-1) / 2$ is simply related to symmetry considerations, i.e. it is the number of couple of points in the correlation function, as conjectured in Ref. [12.

For the comparison with numerical simulations, it is convenient to consider the scaling of the structure functions $\left\langle|\theta(x, t)-\theta(0, t)|^{p}\right\rangle \sim|x|^{\zeta_{p}}$. In particular, the prediction for the fourth and the sixth order are

$$
\zeta_{4}=4-6 \xi+O\left(\xi^{2}\right) \simeq \frac{4}{1+1.5 \xi} \quad \text { and } \quad \zeta_{6}=6-15 \xi+O\left(\xi^{2}\right) \simeq \frac{6}{1+2.5 \xi}
$$

where we have used the classical procedure of Padé approximants [13]. For the comparison we have considered $\xi=0.5$. The measured second-order structure function is presented in Fig. 3. The fourth and sixth order structure functions are shown in Fig. 4. It is worth to note that also low order moments have anomalous scaling. The measured exponents for the moments of order $1 / 2$ and $1 / 8$ are 0.585 and 0.163 . The corresponding normal values are 0.375 and $\simeq 0.094$. It is an interesting issue the relation of these anomalies with those of correlation functions. Note also that $\xi=1$ separates the cases of negatively $(\xi<1)$ and positively $(\xi>1)$ correlated increments of the velocity field. We are currently investigating the influence of positive correlations on structures and anomalous scaling.

In conclusion, we have introduced and investigated a $1 \mathrm{D}$ white-in-time passive scalar model. Numerical simulations show that the typical configuration of the field is strongly structured. The possible role of structures as "atoms" for intermittency has recently been addressed in Ref. [14] for shell models (for white-in-time shell models see Ref. [15]). The conclusion of Ref. [14 was that the single structures are not quite elementary, i.e. 
interactions between them and with the background play a key role for scaling properties. This is also the case for our model. A naif argument based on the scaling of a single structure would indeed suggest the same asymptotic scaling as in Burgers equation, i.e. a constant unit value. Already the second order structure function scales however with an exponent larger than one for $\xi<1$. In the zero mode formalism used here, structures and their correlations do not appear explicitly, but only via their global statistical effects. Our results indicate that these effects are correctly taken into account by perturbative expansions around Gaussian limits. The agreement points in the direction of the scaleinvariant zero mode mechanism for the intermittency of the model.

Acknowledgments. We are grateful to E. Balkovski, B. Dubrulle, G. Falkovich, U. Frisch, I. Kolokolov and V. Yakhot for helpful discussions. We thank the "Meteo-Hydrological Center of Liguria Region" and the "Swiss Scientific Computing Center" where part of the numerical analysis was done.

\section{References}

[1] R.H. Kraichnan, Phys. Rev. Lett., 52, 1016, (1994).

[2] M. Chertkov, G. Falkovich, I. Kolokolov \& V. Lebedev, Phys. Rev. E, 52, 4924 (1995).

[3] K. Gawȩdzki \& A. Kupiainen, Phys. Rev. Lett., 75, 3834, (1995).

[4] B.I. Shraiman \& E.D. Siggia, C.R. Acad. Sci., 321, Série II, 279, (1995).

[5] A.L. Fairhall, O. Gat, V.S. L'vov \& I. Procaccia, Phys. Rev. E, 53, 3518, (1996).

[6] A. Pumir, Europhys. Lett., 34, 25, (1996).

[7] M. Vergassola, Phys. Rev. E, 53, R 3021, (1996).

[8] R.H. Kraichnan, V. Yakhot \& S. Chen, Phys. Rev. Lett., 75, 240, (1995).

[9] P.E. Kloeden \& E. Platen, Numerical Solution of Stochastic Differential Equations, Springer (1992).

[10] W. Feller, An Introduction to Probability Theory and its Applications, Vol. 1, J. Wiley \& Sons, (1950).

[11] D. Bernard, K. Gawȩdzki \& A. Kupiainen, Phys. Rev. E, 54, 2564, (1996). 
[12] M. Chertkov \& G. Falkovich, Phys. Rev. Lett., 76, 2706, (1996).

[13] J. Zinn-Justin, Quantum Field Theory and Critical Phenomena, Oxford Univ. Press, (1989).

[14] J.L. Gilson \& T. Dombre, "Towards a two-fluid picture of intermittency in the GOY model of turbulence", preprint, (1997).

[15] R. Benzi, L. Biferale \& A. Wirth, "Analytic calculation of anomalous scaling in random shell models for passive scalar", preprint, (1997). 